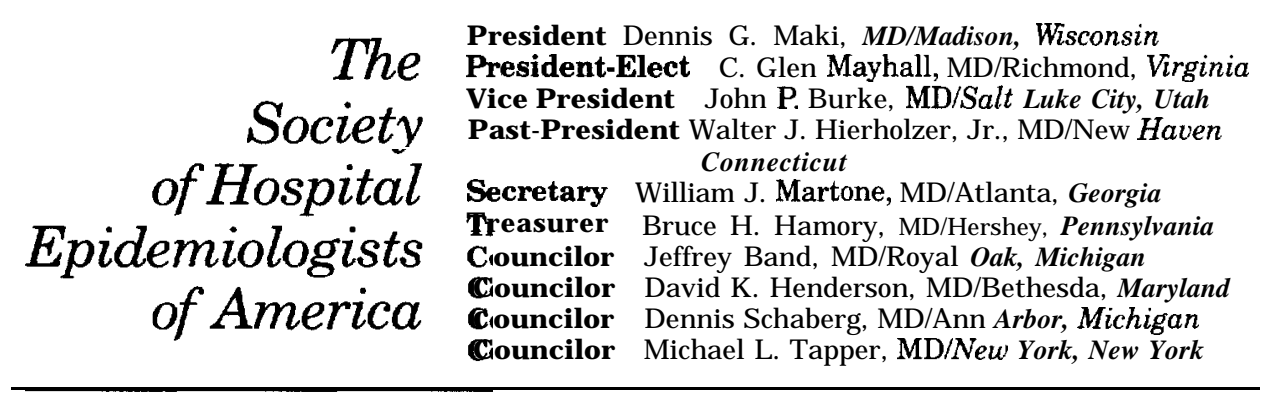

\section{Liaison Report: American Academy of Pediatrics}

Leigh Donowitz, MD, our liaison with the American Academy of Pediatrics Red Book Committee, has had a very active and productive year. After reviewing the Red Book for hospital infection issues, she prepared a chapter on sibling visitation and a statement on employee health activities for the Red Book (many of the specifics regarding employee health are contained in other chapters in the book). These two suggested additions are reproduced below.

\section{SIBLING VISITATION}

Hospital care of pediatric patients, including the well and sick newborn, continues to change. Long-term hospitalizations for chronic and critically ill patients, and birthing centers and shortened postpartum hospitalizations have introduced the sibling onto the wards, into the intensive care unit and into the normal labor, delivery and postpartum environment.

Newborn intensive care units, with their increasing sophistication in medical care and technology, require prolonged hospital stays for the sick newborn, and family visitation has become an important part of neonatal care. Studies to date show that sibling visitation in newborn intensive care units is favorably received by parents and that there is no increase in bacterial colonization or subsequent infection in either the sick or well newborn who has been visited by his or her brothers or sisters. These studies are limited by small numbers, and the concept of sibling visitation needs continued evaluation and reporting of risks.

Strict guidelines for sibling visitation should be established and enforced in an effort to maximize visitation opportunities and minimize the risks of nosocomial spread of contagious pathogens brought into the hospital setting by these little visitors. The following recommendations for sibling visitation should be modified by local nursing, pediatric, obstetric and infectious disease staff to address the specific issues in their own hospital settings.

1. Sibling visitation should be encouraged in both the well nursery and newborn intensive care unit, and for chronically and critically ill children.

2. Prior to visitation, parents should be interviewed by a physician or senior nurse as to the current health of the sibling. No child with fever or symp- toms of an acute illness, such as upper respiratory tract infection, gastroenteritis or dermatitis, should be allowed to visit. Siblings who have been exposed to known communicable diseases (i.e., chickenpox) should not be allowed to visit. Following the interview, the physician or nurse should put a written consent for sibling visitation in the permanent patient record.

3. Visitation should be limited to amounts and periods of time that ensure adequate screening, observation and monitoring of the visitor by the medical and nursing staff

4. The visiting sibling should only visit his or her sibling.

5. Children should carefully wash hands prior to patient contact, most specifically with neonatal and immuncompromised silblings.

6. During the entire visit, sibling activity should be supervised by parents or a responsible adult and limited to the mother's or patient's private room and/or some other designated visitation area.

\section{REFERENCES}

1. Renaud MT. Parental response to family centered maternity care and to the implementation of sibling visits. Milit Med. 1981;146:850.

2. Wranesh BL. The effect of sibling visitation 
on bacterial colonization rates in neonates JOGN Nurs. 1982:11:211

3. Umphenour JH. 'Bacterial colonization in neonates with sibling visitation. JOGN Nurs. 1980;9:73

4. Schwab F. Sibling visting in a neonatal intensive care unit. Pediatrics. 1983:71:835.

5. Yamauchi T. Infection control personnel and policies. In: Donowitz LG, ed. Hospital Acquired Infection in the Pediatric Patient. Baltimore, Md: Williams and Wilkins; 1988.

6. Little GA, et al. Postpartum (neonatal) sibling visitation. Pediatrics. 1985;76:650.

\section{EMPLOYEE HEALTH}

Employee health for personnel working with children is of utmost importance because of the high incidence of communicable disease in children and the high risks for certain pediatric patients of infections carried and trasmitted by adults. Whether or not the employee is an inpatient or outpatient healthcare provider or a child care center employee, preventing the transmission of infectious agents between children and employees remains an important issue.

The employees' risks are increased in pediatric well and sick care, because care of children requires close contact. Children do not, as a rule, practice good personal hygiene, and children have an increased number of infections when compared with adults (an average of six to seven upper respiratory infections per year). Employees at particular or extreme risk of acquiring infections from children are those who are pregnant and immuncompromised. In these situations, infection may have untoward complications for the mother (i.e., influenza, varicella, tuberculosis), the fetus (i.e., parvovirus, cytomegalovirus, rubella) or the immunocompromised patient (i.e., parvovirus, respiratory syncytial virus, herpes simplex, varicella/zoster).

The childhood risks of acquir- ing infections from infected adults are also significant. Mild and severe illness in the adult (viral gastroenteritis, upper respiratory infection, varicella, pertussis, herpes simplex, tuberculosis) can cause severe life-threatenting disease in infants and, specifically, premature infants and immunocompromised children. Children, and particularly infants less than one year old, lack immunity to many common viruses and bacteria. Thus, this highly susceptible population requires care by a healthy uninfected employee. Children at greatest risk of lifethreatening infection acquired from adults are infants less than one year old and children who have heart disease, chronic pulmonary disease or are who immunocompromised.

Children also are at risk of epidemic disease. Their lack of immunity (either primary or secondary), their lack of personal hygienic practices and their close living spaces (playrooms, classrooms, etc.) provide easy transmission of infectious agents introduced into these settings.

Prevention of infection via employee health programs is important. All employees should be interviewed and immunity via history of disease or immunization should be documented for rubella, measles, mumps, pertussis, polio, hepatitis and varicella. Specific employee recommendations for each of these infections are made in that specific topic chapter of this book. Available vaccines should be administered to all nonimmune employees. Institutions where vaccines are not readily available should counsel employees on exposure and the need for furloughing if they are exposed to this infectious agent.

Employees should be screened for tuberculosis, immunocompromised illnesses or other common infections (gastroenteritis, dermatitis, upper respiratory infections) that they may not feel warrant medical attention for themselves but pose a very real threat to their young patients.

The pregnant employee needs to be educated on the real and unfounded concerns regarding pediatric care during pregnancy. Rubella, cytomegalovirus and parvovirus are some of the current concerns for the pregnant employee and are discussed in the specific topic chapters of this book.

Employee education is of paramount importance. Transmission of infectious agents, proper handwashing technique and the potential life-threatening risks to the child if they acquire what are mild infections in adults need to be learned by all healthcare providers of children.

\section{More on the Red Book}

In addition, the Red Book section on human immunodeficiency virus (HIV) and universal precautions discusses use of universal precautions in the context of hospitals in areas with high endemic HIV infection rates. Dr. Donowitz has recommended to the Red Book committee that in light of difficulties in measuring endemic HIV rates as well as hepatitis $B$ rates, universal precautions should be recommended for all hospitals. 